\title{
Beryllium abundances along the evolutionary sequence of the open cluster IC 4651
}

\author{
Rodolfo Smiljanic $^{1,2}$, L. Pasquini ${ }^{2}$, C. Charbonnel ${ }^{3,4}$, and N. Lagarde ${ }^{3}$ \\ ${ }^{1}$ IAG, University of São Paulo, Brazil, ${ }^{2}$ ESO, Germany, \\ email: rsmiljan@eso.org \\ ${ }^{3}$ Geneva Observatory, Switzerland, ${ }^{4}$ LATT, CNRS, Université de Toulouse, France
}

\begin{abstract}
The simultaneous investigation of $\mathrm{Li}$ and $\mathrm{Be}$ in stars is a powerful tool in the study of the evolutionary mixing processes. Here, we present beryllium abundances in stars along the whole evolutionary sequence of the open cluster IC 4651. This cluster has a metallicity of $[\mathrm{Fe} / \mathrm{H}]=+0.11$ and an age of 1.2 or 1.7 Gyr. Abundances have been determined from highresolution, high signal-to-noise UVES spectra using spectrum synthesis and model atmospheres. Lithium abundances for the same stars were determined in a previous work. Confirming previous results, we find that the $\mathrm{Li}$ dip is also a Be dip. For post-main-sequence stars, the Be dilution starts earlier within the Hertzsprung gap than expected from classical predictions, as does the Li dilution. Theoretical hydrodynamical models are able to reproduce well all the observed features.
\end{abstract}

Keywords. Stars: abundances, evolution, rotation - Open clusters and associations: individual: IC 4651

\section{Introduction}

In contradiction with standard stellar evolution models, where convection is the only mechanism driving mixing episodes, field and cluster F- and early G-type stars (including the Sun) deplete Li abundances during the main sequence (Lambert \& Reddy 2004; Sestito \& Randich 2005, and references therein). Different physical mechanisms have been proposed to explain these observations: atomic diffusion, mass loss, rotation-induced mixing, internal gravity waves, or combinations of these (see Charbonnel \& Talon 2008 and references therein).

As $\mathrm{Li}$ and Be burn at different temperatures $\left(2.5 \times 10^{6} \mathrm{~K}\right.$ for $\mathrm{Li}$ and $3.5 \times 10^{6} \mathrm{~K}$ for $\mathrm{Be})$, i.e. at different depths in the stellar interior, they help in constraining the transport mechanisms by performing a stellar tomography. In the study of mixing processes as a function of mass and evolutionary status cluster stars are ideal because they have well defined masses and share the same age and initial chemical composition. We derived Be abundances along the whole evolutionary sequence of the open cluster IC 4651, including solar-type, Li-dip, turn-off, subgiant, and red giant stars. With these new results, we investigate in detail the mixing processes in different stellar masses.

\section{Discussion}

The sample analyzed here is composed of 22 stars; 21 with atmospheric parameters and Li abundances from Pasquini et al. (2004) and 1 from Randich et al. (2002). The spectra have $40 \leqslant \mathrm{~S} / \mathrm{N} \leqslant 100$ (per resolution element) and $\mathrm{R} \sim 45000$.

Be abundances were determined using synthetic spectra and the same codes and line lists used in Smiljanic et al. (2009a). As some of the sample stars are fast rotators, we first carefully modeled the slow-rotating stars and tested the effects in the abundances 

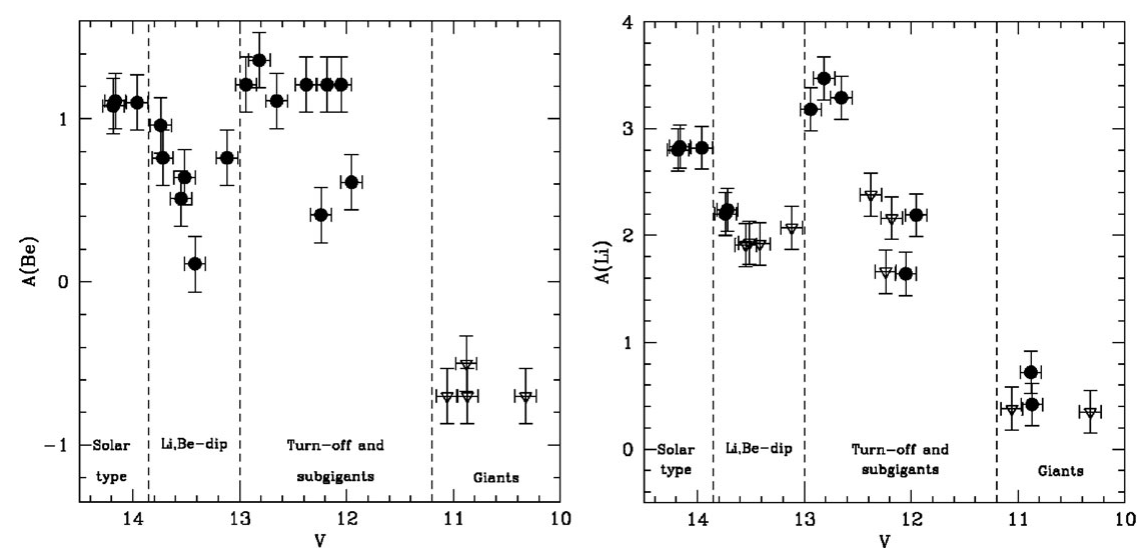

Figure 1. Abundances of Be (left panel) and Li (right panel) as a function of the $\mathrm{V}$ magnitude. Detections are shown as full circles and upper limits as open triangles.

of artificially broadening the spectra (see Smiljanic et al. 2009b for details). Beryllium was detected in all the sample stars except for the giants.

New evolutionary models for stars on the hot side of the dip, including atomic diffusion, meridional circulation, and shear turbulence, were calculated with STAREVOL V3.1 by Lagarde \& Charbonnel (in preparation, see also Charbonnel \& Lagarde this volume) for a range of stellar masses and initial rotation velocities. For stars on the cool side of the Li dip we use the $1.2 \mathrm{M}_{\odot}$ model computed by Talon \& Charbonnel (2005) which has an initial rotation velocity of $50 \mathrm{~km} \mathrm{~s}^{-1}$.

Beryllium abundances are found to follow closely the behavior of the Li abundances (Fig. 1). In a sequence of increasing mass we have first the coolest main-sequence stars that do not present a Be abundance dispersion. This is expected to be due to the impact of internal gravity waves. After that, a well-defined Be dip is seen. This confirms previous results that the Li dip is also a Be dip (Boesgaard \& King 2002, Boesgaard et al. 2004). For post-main-sequence stars we confirm that Be dilution starts earlier than the expected classically. The Be abundances also present a significant dispersion.

The dispersion of $\mathrm{Li}$ and $\mathrm{Be}$ abundances on the blue side of the dip and in evolved stars is very well explained by the models when accounting for a dispersion in the initial values of the stellar rotational velocities. The models reproduce all the $\mathrm{Li}$ and Be features along the CMD of IC 4651. The success in explaining the $\mathrm{Li}$ and Be abundances along the whole evolutionary sequence shows that important steps have been taken towards the proper understanding of the physical mechanisms acting during the stellar evolution.

\section{References}

Boesgaard, A. M., Armengaud, E., \& King, J. R. 2004, ApJ, 605, 864

Boesgaard, A. M. \& King, J. R. 2002, ApJ, 565, 587

Charbonnel, C. \& Talon, S. 2008, in Proceedings of IAUS 252, 163

Lambert, D. L. \& Reddy, B. E. 2004, MNRAS, 349, 757

Pasquini, L., Randich, S., Zoccali, M. et al. 2004, A\&A, 424, 951

Randich, S., Primas, F., Pasquini, L. et al. 2002, A\&GA, 387, 222

Sestito, P. \& Randich, S. 2005, A\&A, 442, 615

Smiljanic, R., Pasquini, L., Bonifacio, P., et al. 2009a, A\&SA, 499, 103

Smiljanic, R., Pasquini, L., Charbonnel, C., \& Lagarde, N. 2009b, arXiv:0910.4399, AESA, in press

Talon, S. \& Charbonnel, C. 2005, A\&A, 440, 981 